PROCEEDINGS OF THE

AMERICAN MATHEMATICAL SOCIETY

Volume 137, Number 9, September 2009, Pages 2953-2957

S 0002-9939(09)09888-8

Article electronically published on April 3, 2009

\title{
A CONSTRUCTIVE BOUND ON KISSING NUMBERS
}

\author{
CHAOPING XING
}

(Communicated by Wen-Ching Winnie Li)

\begin{abstract}
In the present paper, by making use of the concatenation of $17^{2}-1=288$ points on the sphere of radius 4 in $\mathbb{R}^{16}$ and subcodes of algebraic geometry codes over $\mathbb{F}_{17^{2}}$, we improve the best-known constructive bound on kissing numbers by A. Vardy.
\end{abstract}

\section{INTRODUCTION}

One classical question in the geometry of numbers is how many nonoverlapping balls of the same radius in $n$-dimensional Euclidean space $\mathbb{R}^{n}$ can be arranged so that they all just touch, or "kiss", another ball of the same size. This problem is equivalent to packing points on a sphere (i.e., the surface of a ball [1]).

Let us give a precise definition of kissing numbers. The kissing number $K_{n}$ (or Newton number after the originator of the problem) is defined to be the largest number of nonoverlapping balls in $\mathbb{R}^{n}$ so that they all just touch a given ball (all balls here have the same size). The exact values of $K_{n}$ are known only for a few small dimensions $n$. This is equivalent to saying that $K_{n}$ is the size of the largest set of points on a sphere in $\mathbb{R}^{n}$ of radius $r$ such that any two distinct points in the set have distance at least $r$. This motivates us to define spherical codes.

A spherical code $S$ in $\mathbb{R}^{n}$ is a set of points on a sphere $\mathcal{S}_{n}(r)$ of radius $r$ and center $\mathbf{0}$ ( $\mathbf{0}$ denotes the origin) in $\mathbb{R}^{n}$; i.e., $S$ is a subset of

$$
\mathcal{S}_{n}(r):=\left\{\left(x_{1}, \ldots, x_{n}\right) \in \mathbb{R}^{n}: \sqrt{\sum_{i=1}^{n} x_{i}^{2}}=r\right\} .
$$

The distance of $S$ is defined to be

$$
d_{E}(S):=\inf \left\{d_{E}(\mathbf{x}, \mathbf{y}): \mathbf{x} \neq \mathbf{y}, \mathbf{x}, \mathbf{y} \in S\right\}
$$

where $d_{E}(\mathbf{x}, \mathbf{y})$ denotes the Euclidean distance of two points $\mathbf{x}$ and $\mathbf{y}$. Thus, the kissing number is equal to

$$
K_{n}=\max \left\{|S|: S \subset \mathcal{S}_{n}(r), d_{E}(S) \geq r\right\} .
$$

Remark 1.1. (i) Note that $K_{n}$ is independent of the radius $r$.

(ii) Some authors always consider spherical codes in $\mathcal{S}_{n}(1)$ (e.g., see [1, p. 24]). We find that sometimes it is more convenient if the radius is not fixed.

Received by the editors October 20, 2008, and, in revised form, January 9, 2009.

2000 Mathematics Subject Classification. Primary 11H06, 11H31, 05B40, 94B75.

The author was supported by the Singapore MOE Tier 2 grant T208B2206 and the National Scientific Research Project 973 of China 2004CB318000. 
If the distance of $S$ is strictly less than or greater than radius $r$, then we can generalize the definition of kissing numbers in terms of angles.

Let $\phi$ be an angle with $0<\phi<90^{\circ}$ and let $r, \rho$ satisfy $\sin (\phi / 2)=\rho /(2 r)$. Then the kissing number $K_{n}(\phi)$ is defined by

$$
K_{n}(\phi):=\max \left\{|S|: S \subset \mathcal{S}_{n}(r), d_{E}(S) \geq \rho\right\} .
$$

It is clear that $K_{n}=K_{n}\left(60^{\circ}\right)$.

By (1.2), a spherical code $S \subset \mathcal{S}_{n}(r)$ with distance at least $\rho$ gives a lower bound

$$
K_{n}(\phi) \geq|S|
$$

with $\sin (\phi / 2)=\rho /(2 r)$.

In this paper, we are interested in the asymptotic behavior of $K_{n}(\phi)$; i.e., we want to look at how $K_{n}(\phi)$ varies when $n$ tends to infinity for a fixed $\phi$. We define the asymptotic quantity

$$
k(\phi):=\limsup _{n \rightarrow \infty} \frac{\log _{2}\left(K_{n}(\phi)\right)}{n} .
$$

Kabatiansky and Levenshtein [3] show that one has, for $0<\phi<90^{\circ}$,

$$
k(\phi) \lesssim \frac{1+\sin (\phi)}{2 \sin (\phi)} \log _{2}\left(\frac{1+\sin (\phi)}{2 \sin (\phi)}\right)-\frac{1-\sin (\phi)}{2 \sin (\phi)} \log _{2}\left(\frac{1-\sin (\phi)}{2 \sin (\phi)}\right),
$$

and for $0<\phi<\phi^{*}$,

$$
k(\phi) \lesssim-\frac{1}{2} \log _{2}(1-\cos (\phi))-0.0990,
$$

where $\phi^{*} \approx 63^{\circ}$ is the root of the certain equation. In particular, when $\phi=60^{\circ}$, (1.4) gives

$$
k\left(60^{0}\right) \lesssim 0.401 .
$$

On the other hand, a lower bound on $k(\phi)$ is given in [6, 8, by

$$
k(\phi) \geq-\log _{2}(\sin (\phi)) .
$$

This is an existence bound and cannot be constructed even in exponential time. In particular, we obtain

$$
k\left(60^{\circ}\right) \geq-\log _{2}\left(\sin \left(60^{\circ}\right)\right) \approx 0.2075 .
$$

It is shown by A. Vardy (see [1]) that a sequence of spherical codes can be constructed in polynomial time and gives a lower bound

$$
k\left(60^{\circ}\right) \geq \frac{2}{15} \approx 0.13333 .
$$

In this paper, we mainly focus on constructive lower bounds on $k\left(60^{\circ}\right)$. By employing algebraic geometry codes, we give an explicit construction of a spherical code sequence that gives a better constructive bound than (1.7).

Let us describe Vardy's method to get the bound (1.7) without detailed proof.

For an algebraic curve $\mathcal{X}$ over the finite field $\mathbb{F}_{q}$ of $q$ elements, choose $n+1$ distinct $\mathbb{F}_{q}$-rational points $P_{0}, P_{1}, \ldots, P_{n}$ of $\mathcal{X}$. A one-point Goppa geometric code of length $n$ over $\mathbb{F}_{q}$ is defined by

$$
C_{\mathcal{X}}\left(m P_{0} ; P_{1}, P_{2}, \ldots, P_{n}\right):=\left\{\left(f\left(P_{1}\right), f\left(P_{2}\right), \ldots, f\left(P_{n}\right)\right): f \in \mathcal{L}\left(m P_{0}\right)\right\},
$$

where $\mathcal{L}\left(m P_{0}\right)$ is the Riemann-Roch space defined by

$$
\mathcal{L}\left(m P_{0}\right):=\left\{f \in \mathbb{F}_{q}(\mathcal{X})^{*}: \nu_{P_{0}}(f) \geq-m, \nu_{P}(f) \geq 0 \text { for all } P \neq P_{0}\right\} \cup\{0\} .
$$


The code $C_{\mathcal{X}}\left(m P_{0} ; P_{1}, P_{2}, \ldots, P_{n}\right)$ has parameters $[n, k \geq m-g+1, d \geq n-m]$. Moreover, this code can be explicitly constructed as long as the base curve $\mathcal{X}$ is explicitly given.

From [2], there is a family $\{\mathcal{X}\}$ of algebraic curves over $\mathbb{F}_{16^{2}}$ that can be explicitly constructed such that $N(\mathcal{X}) \rightarrow \infty$ and $N(\mathcal{X}) / g(\mathcal{X}) \rightarrow 15$. For each curve $\mathcal{X}$ in this family, let $C_{\mathcal{X}}\left(m P_{0} ; P_{1}, P_{2}, \ldots, P_{n}\right)$ be a one-point Goppa geometric code defined above with $n:=N(\mathcal{X})-1$ and $m:=\lfloor n / 3\rfloor$. Then the Hamming distance $\delta$ of the code is at least $n-m \geq 2 n / 3$.

The binary Preparata code of length 16 (see [4, p.99]) has parameters $\left(16,2^{8}, 6\right)$. We change the $2^{8}=256$ codewords in this code into 256 points on the sphere $\mathcal{S}_{16}(4)$ of radius 4 in $\mathbb{R}^{16}$ by sending 1 to 1 and 0 to -1 . Denote by $S$ the set consisting of these 256 points on $\mathcal{S}_{16}(4)$. Then it is easy to see that $d_{E}(S) \geq$ $\sqrt{4 \times 6}=\sqrt{24}$. Let $\chi$ be a bijection from $\mathbb{F}_{16^{2}}$ to $S$ (such a bijection exists since these two sets have the same size). Then $\left(\chi\left(c_{1}\right), \ldots, \chi\left(c_{n}\right)\right)$ is a point on the sphere $\mathcal{S}_{16 n}(\sqrt{16 n})$ for any codeword $\left(c_{1}, \ldots, c_{n}\right)$ of $C_{\mathcal{X}}\left(m P_{0} ; P_{1}, P_{2}, \ldots, P_{n}\right)$. Thus, the set $\chi\left(C_{\mathcal{X}}\left(m P_{0} ; P_{1}, P_{2}, \ldots, P_{n}\right)\right)$ becomes a spherical code $S_{\mathcal{X}}$ on the sphere $\mathcal{S}_{16 n}(\sqrt{16 n})$ with $d_{E}\left(S_{\mathcal{X}}\right) \geq \sqrt{24 \delta} \geq \sqrt{16 n}$. Hence, we have

$$
K_{16 n} \geq\left|C_{\mathcal{X}}\left(m P_{0} ; P_{1}, P_{2}, \ldots, P_{n}\right)\right| \geq\left(16^{2}\right)^{m-g(\mathcal{X})+1} .
$$

The above lower bound gives the constructive bound (1.7) by letting $N(\mathcal{X}) \rightarrow \infty$.

In this paper, we extend the above method by A. Vardy and obtain the following result.

Theorem 1.2. We have a constructive bound

$$
k\left(60^{\circ}\right) \geq \frac{1}{16}\left(\left(\frac{1}{3}-\frac{1}{16}\right) \log _{2}\left(17^{2}\right)+\log _{2}\left(1-\frac{1}{17^{2}}\right)\right) \approx 0.138065 .
$$

\section{Proof of Theorem 1.2}

Lemma 2.1. Let $\mathcal{X}$ be an algebraic curve over $\mathbb{F}_{q}$ with $n+1$ distinct $\mathbb{F}_{q}$-rational points $P_{0}, P_{1}, \ldots, P_{n}$. Let $g$ be the genus of $\mathcal{X}$. If $m$ is an integer satisfying $m>$ $2 g+2$, then

$$
\left|\mathcal{L}\left(m P_{0}\right) \backslash \bigcup_{i=1}^{n} \mathcal{L}\left(m P_{0}-P_{i}\right)\right| \geq q^{m-g+1}\left(1-\frac{1}{q}\right)^{n}-n 2^{n} q^{g+1} .
$$

Proof. Let $\ell=2\lfloor m / 2-g\rfloor+1 \leq m-2 g+1$. By the inclusion and exclusion principle, we have

$$
\begin{aligned}
\bigcup_{i=1}^{n} \mathcal{L}\left(m P_{0}-P_{i}\right) \mid & =\sum_{k=1}^{n}(-1)^{k+1} \sum_{i_{1}, \ldots, i_{k}}\left|\bigcap_{j=1}^{k} \mathcal{L}\left(m P_{0}-P_{i_{j}}\right)\right| \\
& =\sum_{k=1}^{n}(-1)^{k+1} \sum_{i_{1}, \ldots, i_{k}}\left|\mathcal{L}\left(m P_{0}-\sum_{j=1}^{k} P_{i_{j}}\right)\right| \\
& \leq \sum_{k=1}^{\ell}(-1)^{k+1} \sum_{i_{1}, \ldots, i_{k}}\left|\mathcal{L}\left(m P_{0}-\sum_{j=1}^{k} P_{i_{j}}\right)\right| \\
& =\sum_{k=1}^{\ell}(-1)^{k+1}\left(\begin{array}{l}
n \\
k
\end{array}\right) q^{m-k-g+1} \text { (by Riemann-Roch Theorem) }
\end{aligned}
$$


Hence, we have

$$
\begin{aligned}
\left|\mathcal{L}\left(m P_{0}\right) \backslash \bigcup_{i=1}^{n} \mathcal{L}\left(m P_{0}-P_{i}\right)\right| & \geq q^{m-g+1}-\sum_{k=1}^{\ell}(-1)^{k+1}\left(\begin{array}{l}
n \\
k
\end{array}\right) q^{m-k-g+1} \\
& =q^{m-g+1}\left(1-\frac{1}{q}\right)^{n}+\sum_{k=\ell+1}^{n}(-1)^{k+1}\left(\begin{array}{l}
n \\
k
\end{array}\right) q^{m-k-g+1} \\
& \geq q^{m-g+1}\left(1-\frac{1}{q}\right)^{n}-n 2^{n} q^{m-\ell-g} \\
& \geq q^{m-g+1}\left(1-\frac{1}{q}\right)^{n}-n 2^{n} q^{g+1}
\end{aligned}
$$

Note that in the above inequalities we used the facts that $\left(\begin{array}{l}n \\ k\end{array}\right) \leq 2^{n}$ and $2 g-1 \leq$ $m-\ell \leq 2 g+1$.

Remark 2.2. If the curve $\mathcal{X}$ is explicitly given, then both $\mathcal{L}\left(m P_{0}\right)$ and $\mathcal{L}\left(m P_{0}-\right.$ $\left.P_{i}\right)$ can be explicitly constructed in polynomial time. Thus, the set $\mathcal{L}\left(m P_{0}\right) \backslash$ $\bigcup_{i=1}^{n} \mathcal{L}\left(m P_{0}-P_{i}\right)$ can be explicitly constructed as well.

Proof of Theorem 1.2 , Let $\{\mathcal{X}\}$ be a family of curves over $\mathbb{F}_{17^{2}}$ that is explicitly given in 2] such that $N(\mathcal{X}) \rightarrow \infty$ and $N(\mathcal{X}) / g(\mathcal{X}) \rightarrow 16$. For each curve $\mathcal{X}$ in this family, consider a code over $\mathbb{F}_{17^{2}} \backslash\{0\}$ defined by

$$
C_{\mathcal{X}}:=\left\{\left(f\left(P_{1}\right), f\left(P_{2}\right), \ldots, f\left(P_{n}\right)\right): f \in \mathcal{L}\left(m P_{0}\right) \backslash \bigcup_{i=1}^{n} \mathcal{L}\left(m P_{0}-P_{i}\right)\right\},
$$

where $n=N(\mathcal{X})-1, m=\lfloor n / 3\rfloor$ and $P_{0}, P_{1}, \ldots, P_{n}$ are $n+1$ distinct $\mathbb{F}_{q}$-rational points on $\mathcal{X}$. Then it is easy to see that $\left(f\left(P_{1}\right), f\left(P_{2}\right), \ldots, f\left(P_{n}\right)\right) \neq\left(g\left(P_{1}\right), g\left(P_{2}\right)\right.$, $\ldots, g\left(P_{n}\right)$ ) for two distinct functions $f, g$ in $\mathcal{L}\left(m P_{0}\right)$ since $m<n$ (see [7, [5]). Moreover, the Hamming distance of $C_{\mathcal{X}}$ is at least $n-m \geq 2 n / 3$.

From the previous section, we know that there is a spherical code $S$ on the sphere $\mathcal{S}_{16}(4)$ with $|S|=2^{8}=256$ and $d_{E}(S) \geq \sqrt{24}$ such that all coordinates in each codeword are equal to 1 or -1 . We now add 32 points $( \pm 4,0, \ldots, 0),(0, \pm 4,0, \ldots, 0)$, $\ldots,(0, \ldots, 0, \pm 4)$ on $\mathcal{S}_{16}(4)$ to the set $S$ to get a spherical code $S^{\prime}$. Then it is easy to see that $S^{\prime} \subset \mathcal{S}_{16}(4),\left|S^{\prime}\right|=|S|+32=17^{2}-1$ and $d_{E}\left(S^{\prime}\right)=\sqrt{24}$. Through a bijection $\tau$ from $\mathbb{F}_{17^{2}} \backslash\{0\}$ to $S^{\prime}$, the set $\tau\left(C_{\mathcal{X}}\right)$ is a spherical code on $\mathcal{S}_{16 n}(\sqrt{16 n})$. Moreover, we have

$$
d_{E}\left(\tau\left(C_{\mathcal{X}}\right)\right) \geq \sqrt{24(n-m)} \geq \sqrt{16 n} .
$$

Hence, we have

$$
K_{16 n} \geq\left|C_{\mathcal{X}}\right| \geq q^{m-g+1}\left(1-\frac{1}{q}\right)^{n}-n 2^{n} q^{g+1}
$$

with $q=17^{2}$.

Considering the limit limsup $\sup _{n \rightarrow \infty} \log _{2}\left(K_{16 n}\right) /(16 n)$ for $K_{16 n}$ from the inequality (2.2), we obtain the desired result. 


\section{REFERENCES}

[1] J. H. Conway and N. J. A. Sloane, "Sphere Packings, Lattices and Groups", Springer-Verlag, New York, 1999 (Third Edition). MR 1662447 (2000b:11077)

[2] A. Garcia and H. Stichtenoth, A tower of Artin-Schreier extensions of function fields attaining the Drinfel' $d$-Vlădut bound, Invent. Math., 121 (1995), 211-222. MR.1345289(96d:11074)

[3] G. A. Kabatianskiǔ and V. I. Levenšteĭn, Bounds for packings on the sphere and in space, Problemy Peredachi Informatsii 14, No. 1 (1978), 3-25. MR0514023 (58:24018)

[4] S. Ling and C. P. Xing, "Coding Theory. A First Course", Cambridge University Press, Cambridge, 2004. MR2048591 (2005c:94001)

[5] H. Niederreiter and C. P. Xing, "Rational Points on Curves over Finite Fields: Theory and Applications", London Math. Soc. Lecture Note Series 285, Cambridge University Press, Cambridge, 2001. MR.1837382(2002h:11055)

[6] C. E. Shannon, Probability of error for optimal codes in a Gaussian channel, Bell System Technical Journal, 38 (1959), 611-656. MR0103137 (21:1920)

[7] M. A. Tsfasman and S. G. Vlăduț, "Algebraic-Geometric Codes", Kluwer, Dordrecht, 1991. MR.1186841 (93i:94023)

[8] A. D. Wyner, Capabilities of bounded discrepancy decoding, Bell System Technical Journal, 44 (1965), 1061-1122. MR0180417 (31:4652)

Division of Mathematical Sciences, School of Physical and Mathematical Sciences, Nanyang Technological University, Singapore 637371, Republic of Singapore

E-mail address: xingcp@ntu.edu.sg 